\title{
Terlipressin Induced Reversible Valvular Dysfunction in a Cirrhotic Patient for Living Donor Liver Transplant: Case Report
}

\section{Manoj Gupta* and K R Vasudevan}

Department of Liver Transplant and Surgical Gastroenterology, PSRI Hospital, New Delhi, India

*Corresponding Author: Manoj Gupta, Department of Liver Transplant and Surgical Gastroenterology, PSRI Hospital, New Delhi, India.
Received: June 07, 2021

Published: July 13, 2021

(C) All rights are reserved by Manoj Gupta and K R Vasudevan.

\begin{abstract}
Terlipressin, a vasopressin analogue, has known cardiac side effects e.g. arrhythmias, reduced left ventricular ejection fraction, non ST elevation myocardial infarction. However, to the best of our knowledge reversible valvular insufficiency with pulmonary hypertension with terlipressin has never been described in the literature in cirrhotics. As in the present case it was reversible therefore definitive treatment i.e. liver transplantation should not be deferred in this subgroup of patients.
\end{abstract}

Keywords: Terlipressin; Arginine Vasopressin; Myocardial Infarction; Arrhythmias

\section{Introduction}

Terlipressin, a synthetic analogue of arginine vasopressin, is widely used for treating cirrhosis related complications like variceal bleeding, hepatorenal syndrome, portopulmonary hypertension. Though it has shown promising results in the management of these complications it has some known side effects e.g. cardiac arrhythmias, hypertension, loose motions and abdominal pain. To the best of our knowledge, reversible valvular insufficiency and pulmonary hypertension with terlipressin has not been described earlier in the literature.

\section{The Case}

A 55 years old female was admitted to our centre as a case of hepatitis $\mathrm{C}$ related cirrhosis decompensated with jaundice, ascites and hepatorenal syndrome. Her CTP score was 11 and MELD score was 37. At the time of admission her serum creatinine was $5.7 \mathrm{mg} /$ dl. She was started on inj. terlipressin (dosage) with albumin. Ascitic fluid analysis was suggestive of spontaneous bacterial peritonitis (cell count, differential count, cultures). Broad spectrum antibiotics were started according to the culture and sensitivity reports. She was advised living donor liver transplantation (LDLT) and preoperative work up was started. On 2D echocardiography no anatomical or valvular abnormality was identified and right and left systolic function was normal with left ventricular ejection fraction of $65 \%$ and pulmonary artery systolic pressure of 23 .

Initially some improvement in serum creatinine was seen it again started rising and therefore the dose of terlipressin was gradually increased to $2 \mathrm{mg}$ three times a day. After 5 days of treatment, her urine output increased and s. creatinine improved to $1.15 \mathrm{mg} / \mathrm{dl}$. She complained of breathlessness 5 days after increasing the dose to $6 \mathrm{mg} /$ day. 2D echocardiography showed severe mitral regurgitation (jet area (JA) $10.9 \mathrm{~cm}^{2}$ ), mild tricuspid regurgitation, PASP $54 \mathrm{~mm} \mathrm{Hg}$, moderate PAH and LVEF 64\%. Troponin I was within normal limits (0.04). Sildenafil $40 \mathrm{mg} /$ day was started, but her pulmonary artery pressures remained high and MR persisted on echocardiography despite sildenafil therapy for 2 days. A suspicion of drug induced MR and PAH was made and terlipressin was stopped. A repeat echocardiography after 3 days of terlipressin withdrawal revealed traces MR and a PASP of 33mmHg. But her renal functions again worsened (S. creatinine- 1.95). In view 
of worsening hepatorenal syndrome and stable cardiac function a decision to proceed with liver transplantation was made. She underwent orthotopic living donor liver transplantation. Intraoperatively she remained stable and maintained good urine output. Her S. creatinine on post-operative day 1 was 1.63 , which progressively improved to 1.08 on post-operative day 5 .
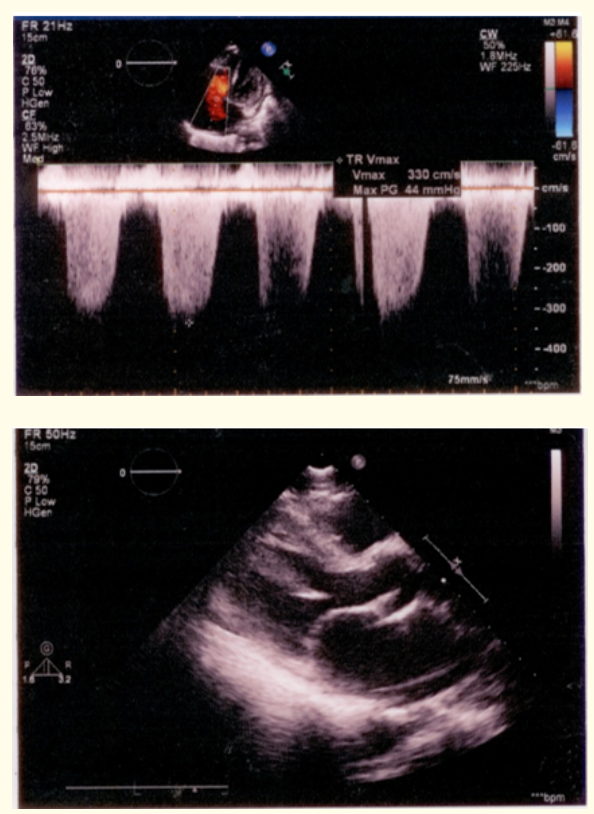

Figure 1: Echocardiogram after starting Terlipressin showing severe MR (Jet area $10.9 \mathrm{~cm} 2$ ) with moderate PAH (PASP $54 \mathrm{~mm}$ $\mathrm{Hg}$ ).

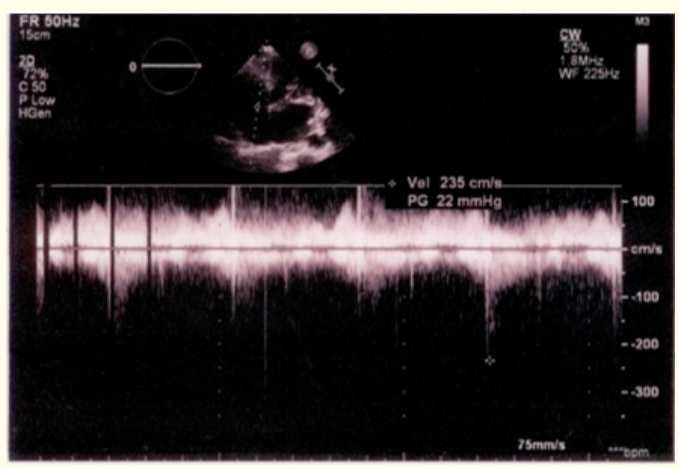

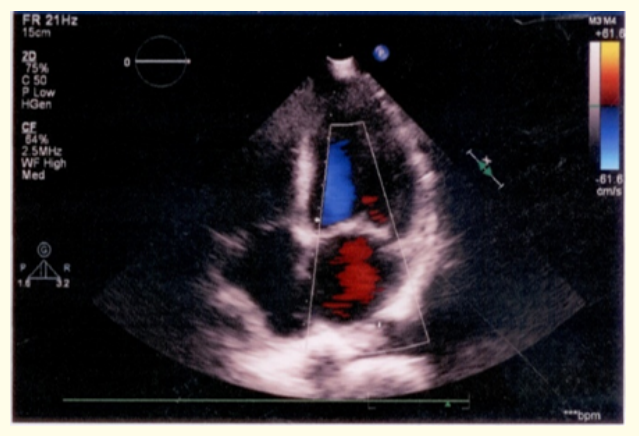

Figure 2: Echocardiogram after stopping Terlipressin showing trace MR with PASP $33 \mathrm{~mm} \mathrm{Hg}$.

\section{Discussion and Conclusion}

Terlipressin is commonly used in the treatment of the hepatorenal syndrome (HRS) and bleeding oesophageal varices [1-3]. Cardiovascular side-effects of Terlipressin are cyanosis, ischemia of extremities, cardiac arrhythmias, hypertension, angina pectoris, and myocardial infarction [4-6].

Naser., et al. first reported a case describing the association between intravenous terlipressin and cardiac effects like non-STelevation myocardial infarction, severe myocardial dysfunction in a patient with upper GI bleeding [7].

Aleksander Kraga reported that in advanced cirrhosis, the increase in after load and end diastolic volume after terlipressin administration results in a decrease in left ventricle wall motion and ejection fraction but myocardial perfusion remain preserved. One animal study showed that terlipressin only affects coronary blood flow in supratherapeutic levels [8].

Our patient had mitral valvular regurgitation on high doses of terlipressin which reversed after stoppage of the drug. Though her renal functions deteriorated after discontinuation of drug, liver transplantation was planned for advanced cirrhosis since her cardiac functions stabilized.

Though terlipressin has been reported to decrease PAP in cirrhotic patients with mild pulmonary hypertension [9], we noticed deterioration of pulmonary hypertension on higher doses of terli- 
pressin which could be due of mitral valvular regurgitation, which reversed on withdrawal of the drug.

Only few case reports and observations from randomized studies have indicated that ischemic side-effects of terlipressin related to the heart may occur [4-6], but the reversible valvular dysfunction has never been reported.

This case report highlights the important cardiac side effect in the form of valvular dysfunction on high dose of terlipressin. Considering the reversibility of this effect, definitive therapeutic option of end stage liver disease e.g. liver transplant should not be deferred in this subset of patients.

\section{Bibliography}

1. Ioannou G., et al. "Terlipressin for acute oesophageal variceal haemorrhage". Cochrane Database of Systematic Reviews 1 (2003): CD002147.

2. Moreau R and Lebrec D. "The use of vasoconstrictors in patients with cirrhosis: type 1 HRS and beyond". Hepatology 43 (2006): 385-394.

3. Salerno F., et al. "Diagnosis, prevention and treatment of hepatorenal syndrome in cirrhosis". Gut 56 (2007): 1310-1318.

4. Krag A., et al. "Efficacy and safety of terlipressin in cirrhotic patients with variceal bleeding or hepatorenal syndrome". Advances in Therapy 25 (2008): 1105-1140.

5. Rosario R., et al. "Myocardial infarction after injection of terlipressin for digestive hemorrhage". Gastroentérologie Clinique et Biologique 20 (1996): 712-713.

6. Lee MY., et al. "Terlipressin-related acute myocardial infarction: a case report and literature review". Kaohsiung Journal of Medical Sciences 20 (2004): 604-608.

7. Elzouki Abdul-Naser., et al. "Terlipressin-induced severe left and right ventricular dysfunction in patient presented with upper gastrointestinal bleeding: case report and literature review". American Journal of Emergency Medicine 28 (2010): 540.e1-540.e6.

8. Aleksander Krag., et al. "Effects of a single terlipressin administration on cardiac function and perfusion in cirrhosis". European Journal of Gastroenterology and Hepatology 22.9 (2010).
9. Altintas E., et al. "Effects of terlipressin on systolic pulmonary artery pressure of patients with liver cirrhosis". World Journal of Gastroenterology 10 (2004): 2278-2280.

Volume 4 Issue 8 August 2021

(C) All rights are reserved by Manoj Gupta and $\mathrm{K} R$ Vasudevan. 